\title{
World Journal of Pediatric Surgery \\ Validation of the Japan Coma Scale for the prediction of mortality in children: analysis of a nationwide trauma database
}

Yuki Enomoto (D) ,, ${ }^{1,2}$ Yusuke Tsutsumi, ${ }^{2}$ Asuka Tsuchiya, ${ }^{3,4}$ Takahiro Kido, ${ }^{5}$ Koji Ishigami, ${ }^{2}$ Masahito Togo, ${ }^{2}$ Susumu Yasuda, ${ }^{2}$ Yoshiaki Inoue ${ }^{1}$

To cite: Enomoto Y, Tsutsumi Y, Tsuchiya A, et al. Validation of the Japan Coma Scale for the prediction of mortality in children: analysis of a nationwide trauma database. World Jnl Ped Surgery 2022;5:e000350. doi:10.1136/ wjps-2021-000350

- Additional supplemental material is published online only. To view, please visit the journal online (http://dx.doi.org/10.1136/ wjps-2021-000350).

Received 15 August 2021 Accepted 10 December 2021

\section{Check for updates}

(c) Author(s) (or their employer(s)) 2022. Re-use permitted under CC BY-NC. No commercial re-use. See rights and permissions. Published by BMJ.

${ }^{1}$ Department of Emergency and Critical Care Medicine, University of Tsukuba, Tsukuba, Ibaraki, Japan

${ }^{2}$ Department of Emergency and Critical Care Medicine, Mito Medical Center, Mito, Ibaraki, Japan

${ }^{3}$ Department of Emergency and Critical Care Medicine, Tokai University School of Medicine Graduate School of Medicine, Isehara, Kanagawa, Japan ${ }^{4}$ Department of Clinical Epidemiology and Health Economics, The University of Tokyo, Bunkyo-ku, Tokyo, Japan ${ }^{5}$ Department of Pediatrics, University of Tsukuba Hospital, Tsukuba, Ibaraki, Japan

Correspondence to Dr Yuki Enomoto; enomoto-y@ md.tsukuba.ac.jp

\section{ABSTRACT}

Objective The Japan Coma Scale (JCS) is widely used in clinical practice to evaluate levels of consciousness in Japan. There have been several studies on the usefulness of JCS in adults. However, its usefulness in evaluating children has not been reported. Therefore, this study aimed to assess the usefulness of the JCS for the prediction of mortality in children.

Methods This is a multicenter cohort study which used data from a national trauma registry (Japan Trauma Data Bank). This study included patients under 16 years of age who were treated between 2004 and 2015.

The primary outcome measure was in-hospital mortality. Two models were used to examine each item of the Glasgow Coma Scale (GCS) and the JCS. Model A included the discrete levels of each index. In model B, data regarding age, sex, vital signs on arrival to hospital, the Injury Severity Score, and blunt trauma were added to each index. The effectivity of the JCS score was then evaluated using the area under the curve (AUC) for discrimination, a calibration plot, and the Hosmer-Lemeshow test for calibration.

Results A total of 9045 patients were identified. The AUCs of the GCS and JCS were 0.929 (95\% confidence interval (Cl) 0.904 to 0.954$)$ and $0.930(95 \% \mathrm{Cl} 0.906$ to 0.954$)$ in model $\mathrm{A}$ and 0.975 (95\% $\mathrm{Cl} 0.963$ to 0.987$)$ and 0.974 $(95 \% \mathrm{Cl} 0.963$ to 0.985$)$ in model B, respectively. The results of the Hosmer-Lemeshow test were $0.00(p=1.00)$ and $0.00(p=1.00)$ in model $A$ and $4.14(p=0.84)$ and 8.55 $(p=0.38)$ in model B for the GCS and JCS, respectively. Conclusions We demonstrated that the JCS is as valid as the GCS for predicting mortality. The findings of this study indicate that the JCS is a useful and relevant tool for pediatric trauma care and future research.

\section{INTRODUCTION}

Neurological status is an important predictive indicator in trauma. The Glasgow Coma Scale (GCS) is used globally to assess the severity of brain dysfunction and is a component of several predictive scores. ${ }^{1-5}$ The GCS score has been reported as an important predictive factor for prognosis, even in children. ${ }^{6-8}$

\section{Key messages}

What is already known about this subject?

- In the treatment of pediatric trauma, it is important to determine the level of consciousness.

- The Japan Coma Scale (JCS) is a simple tool for assessing the state of consciousness and is widely used in Japan.

- Although several reports have proven the usefulness of the JCS in adults, its usefulness in examining children has not been reported.

\section{What are the new findings?}

- There were no clinically meaningful differences between the JCS and Glasgow Coma Scale (GCS) in discrimination of in-hospital mortality in pediatric trauma patients.

- The calibration of the JCS was comparable to the GCS in predicting mortality in pediatric trauma cases.

- In children, the JCS and GCS are both valid predictors of mortality.

\section{How might it impact on clinical practice in the} foreseeable future?

- The JCS is a valuable and relevant tool for pediatric trauma care and future research.

The Japan Coma Scale (JCS), which was developed in $1974,{ }^{9}$ is widely used in clinical practice, including in emergency medicine, in Japan, to assess the level of consciousness. Both the JCS and GCS are commonly used in Japan; however, nurses and paramedics ${ }^{10}$ tend to use the JCS because it is easy to score. Healthcare professionals, including physicians, often use the JCS to assess a child's state of consciousness. ${ }^{11}$ Therefore, the JCS is used to assess consciousness in various settings in Japan, such as in prehospital care, ${ }^{10}$ and it is also used in nationwide databases, such as the Nationwide Claims Database ${ }^{1213}$ and the Japan Trauma Data Bank (JTDB). ${ }^{10}{ }^{14}$ Several studies have described the usefulness of the 


\begin{tabular}{|c|c|}
\hline & $\mathrm{N}=9045$ \\
\hline \multicolumn{2}{|c|}{ Glasgow Coma Scale score* } \\
\hline 15 & $6239(69.0)$ \\
\hline 14 & $940(10.4)$ \\
\hline 13 & $380(4.2)$ \\
\hline 12 & $202(2.2)$ \\
\hline 11 & $199(2.2)$ \\
\hline 10 & $183(2.0)$ \\
\hline 9 & $154(1.7)$ \\
\hline 8 & $155(1.7)$ \\
\hline 7 & $177(2.0)$ \\
\hline 6 & $146(1.6)$ \\
\hline 5 & $43(0.5)$ \\
\hline 4 & $60(0.7)$ \\
\hline 3 & $167(1.8)$ \\
\hline \multicolumn{2}{|c|}{ Japan Coma Scale score* } \\
\hline Alert & $5600(61.9)$ \\
\hline $\mid-1$ & $1124(12.4)$ \\
\hline $\mathrm{I}-2$ & $330(3.6)$ \\
\hline $1-3$ & $330(3.6)$ \\
\hline II-10 & $571(6.3)$ \\
\hline II-20 & $177(2.0)$ \\
\hline II-30 & $177(2.0)$ \\
\hline$|I|-100$ & $316(3.5)$ \\
\hline III-200 & $229(2.5)$ \\
\hline III-300 & $191(2.1)$ \\
\hline Age $(y) \dagger$ & $9(5-12)$ \\
\hline \multicolumn{2}{|l|}{ Age group $(y)^{\star}$} \\
\hline $0-1$ & $338(3.7)$ \\
\hline $1-2$ & $745(8.2)$ \\
\hline $3-5$ & 1194 (13.2) \\
\hline $6-9$ & 2891 (32.0) \\
\hline $10-12$ & $1748(19.3)$ \\
\hline $12-17$ & 2129 (23.5) \\
\hline $\operatorname{Sex}(F)^{*}$ & $2732(30.2)$ \\
\hline Hypotension* & $234(2.7)$ \\
\hline Bradypnea* & $917(10.1)$ \\
\hline Tachypnea* & 2344 (25.9) \\
\hline Injury Severity Score† & $9(5-16)$ \\
\hline Blunt trauma* & 8827 (98.3) \\
\hline Mortality* & $162(1.8)$ \\
\hline
\end{tabular}

*Data were presented as $\mathrm{n}(\%)$.

†Data were presented as median (IQR).

$F$, female; IQR, interquartile range.

JCS for the prediction of mortality in adults ${ }^{15-19}$; however, the usefulness of the JCS score in the assessment of pediatric trauma patients has not yet been reported.

Identifying the usefulness of the widely used JCS score would be helpful in assuring the quality of practice and

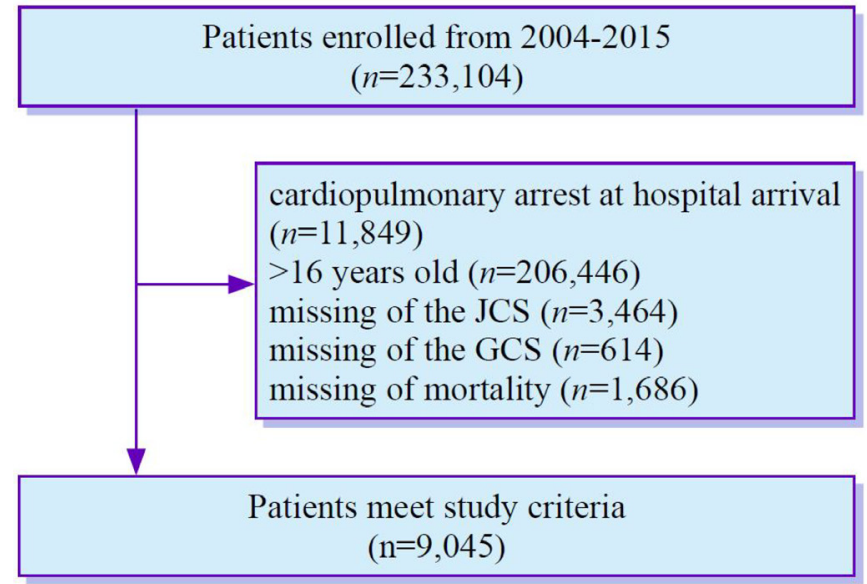

Figure 1 Flow chart of participant selection. GCS, Glasgow Coma Scale; JCS, Japan Coma Scale.

facilitating future research. Therefore, the purpose of this study was to evaluate the usefulness of the JCS in predicting mortality in pediatric trauma patients.

\section{METHODS}

Patient and public involvement

This study was conducted using secondary data and did not involve patients or the public.

\section{Study design and data source}

This was a retrospective cohort study conducted using the National Trauma Database. We obtained the data from the JTDB, which was a joint organization by the Japanese Association for the Surgery of Trauma (Trauma Registry Committee) and the Japanese Association for Acute Medicine (Committee for Clinical Care Evaluation) in 2003. Two hundred and seventy-two hospitals voluntarily submitted data to the JTDB. ${ }^{14}$ Approximately $74 \%$ of all tertiary-level emergency hospitals in Japan have contributed to the database. ${ }^{14}$ To maintain data quality, those who submitted Abbreviated Injury Scale (AIS) scores received training for AIS code. The JTDB registration criteria are usually based on an AIS score of $\geq 3$. Data cleansing was also done by the JTDB. The database contains data on the demographics of each patient, vital signs recorded at the trauma scene and on arrival at the emergency department, pre-existing medical conditions, nature of injury, diagnosis, severity of the injury, surgical and interventional procedures, and patient disposition. Diagnoses for injury were recorded based on the AIS. Severity of anatomical injuries were assessed using the Injury Severity Score (ISS). The state of consciousness on admission was assessed using both the JCS and GCS scores.

\section{Japan Coma Scale}

The development of the JCS in Japan coincided with the publication of the GCS. ${ }^{19}$ Since then, the JCS has been used as a standard scale for assessing consciousness in 
A model A

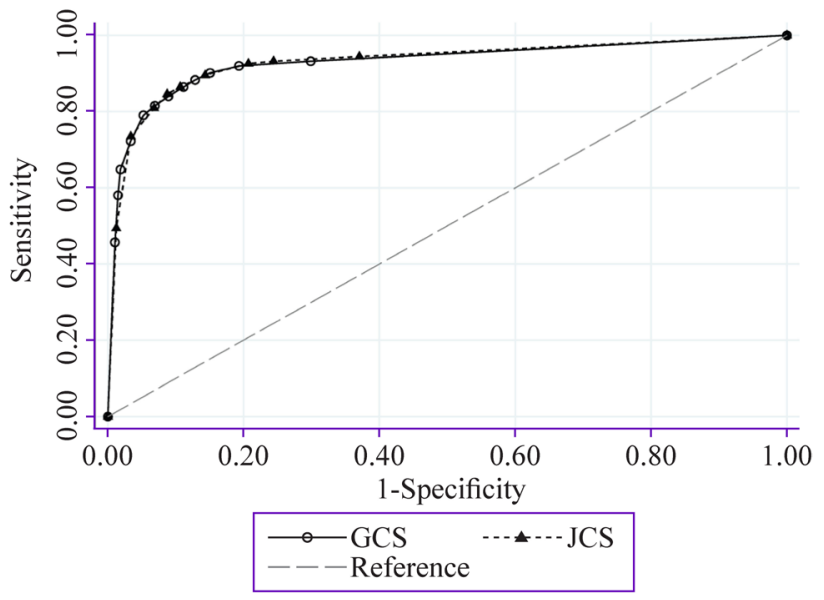

B model B

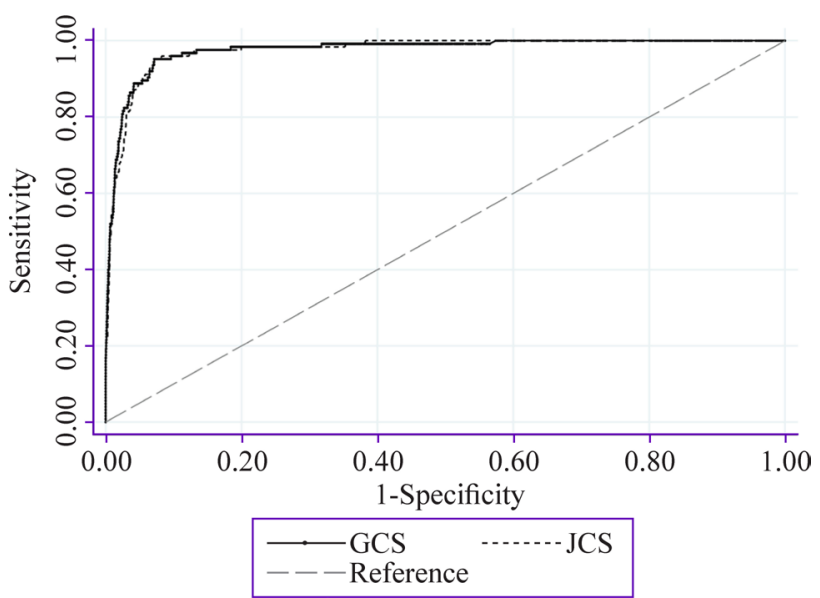

Figure 2 Receiver operating curves of the Glasgow Coma Scale (GCS) and Japan Coma Scale (JCS). (A) Model A; (B) model B.

trauma patients by paramedics and healthcare providers throughout Japan.

The scale consists of four main categories: 0-digit, 1-digit, 2-digit, and 3-digit codes, which correspond to 'alert', 'awake without stimulation', 'awake on stimulation but asleep when stimulation stops', and 'does not wake on any stimulation', respectively. The 1-digit, 2-digit, and 3-digit codes are further divided into three subcategories, making a total of 10 grades overall. The 1-digit code consists of grades 1,2 , and 3, which denote 'almost fully conscious but not normal', 'unable to recognize time, place, or person', and 'unable to recall name or date of birth', respectively. The 2-digit code consists of grades 10, 20, and 30, which denote 'arousable by being spoken to', 'arousable by loud voice', and 'arousable only by repeated mechanical stimuli', respectively. The 3-digit code consists of grades 100, 200, and 300, which denote 'unarousable but responds to avoid the stimuli'; 'unarousable but responds with slight movements, including decerebrate or decorticate postures'; and 'does not respond at all', respectively ${ }^{91620}$ (online supplemental table 1, left row).

Similar to the GCS, the JCS has a modified version for infants. ${ }^{20}{ }^{21}$ In this modified version, the 1-digit code consists of grades 1, 2, and 3, which represent 'laughs when amused, but not fully and aloud', 'does not laugh when amused but able to make eye contact', and 'does not make eye contact with the mother', respectively. The 2-digit code consists of grades 10, 20, and 30, which indicate 'tries to drink something if showed it or wanting and sucking a nipple if showed it', 'opens and turns eyes to the person who calls out', and 'slightly opens eyes after repeated calls', respectively. The 3-digit code consists of grades 100, 200, and 300, which denote 'unarousable but responds to avoid the stimuli'; 'unarousable but responds with slight movements, including decerebrate or decorticate postures'; and 'does not respond at all', respectively (online supplemental table 1, right row). Generally, children older than infants are designated a JCS score using the same criteria used for adults.

\section{Study participants}

We included patients younger than 16 years treated between 2004 and 2015. Patients who were in cardiac arrest at the time of hospital arrival and patients missing data on JCS or GCS scores at the time of hospital arrival or death were excluded.

\section{Variables}

We defined the GCS as an index test. The data collected included data on age, sex, vital signs on arrival at the hospital, the ISS, and blunt trauma. Vital signs were classified as normal, above normal, or below normal in accordance with age. ${ }^{22}$ For details regarding vital signs, please see online supplemental table 2. The main outcome measure was in-hospital mortality.

Physicians assessed the consciousness condition of a patient on arrival at the hospital using the JCS and GCS. In Japan, physicians are trained to use both the GCS and JCS. Each of the GCS and JCS was examined in two models. The first model (model A) included the discrete levels of each index; the second model (model B) included these levels plus seven additional predictors of mortality: age, sex, classified vital signs (hypotension, tachypnea, and bradypnea), the ISS, and blunt trauma. We selected these items based on the Trauma and Injury Severity Score, a common score used for the prediction of mortality due to trauma. ${ }^{3}$

\section{Statistical analyses}

First, the GCS models were validated. The area under the receiver operating characteristic curve (AUC) was computed with a $95 \%$ confidence interval (CI) using 1000 bootstrap replicates. The calibration of the GCS models with a calibration plot was graphically assessed and examined using the Hosmer-Lemeshow test. A $p$ value $<0.05$ indicates the lack of a good fit for the model. ${ }^{23}$ Second, the clinical importance of 
A Glasgow Coma Scale (model A)

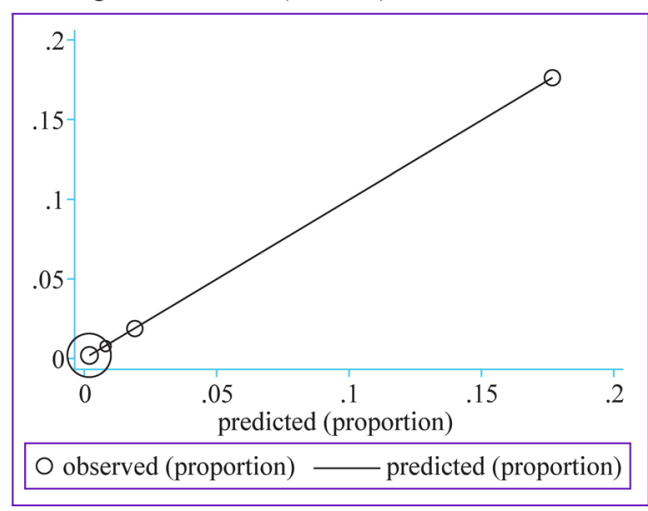

C Glasgow Coma Scale (model B)

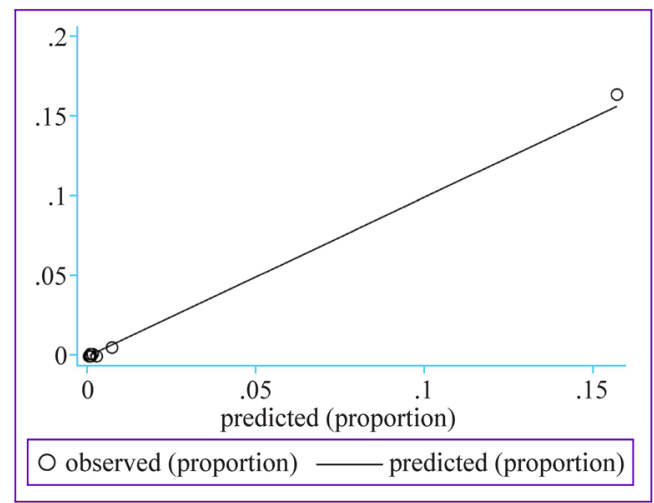

B Japan Coma Scale (model A)

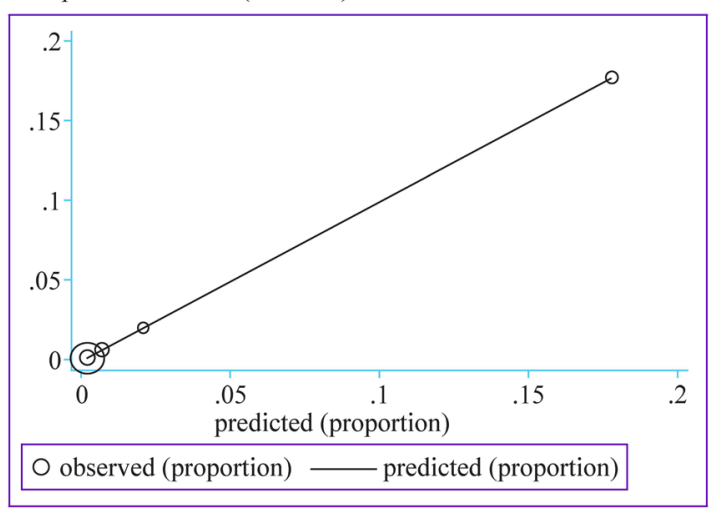

D Japan Coma Scale (model B)

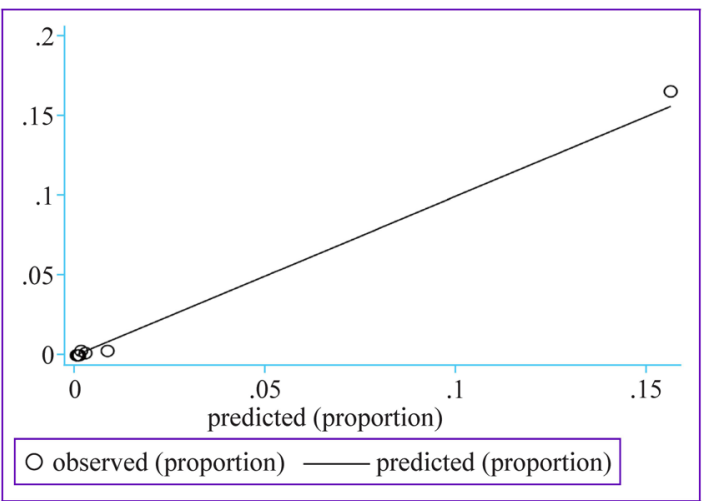

Figure 3 Calibration plot of the Glasgow Coma Scale and Japan Coma Scale. (A) Glasgow Coma Scale (model A). (B) Japan Coma Scale (model A). (C) Glasgow Coma Scale (model B). (D) Japan Coma Scale (model B). The size of each circle represents the number of patients in each stratum.

the JCS was examined. Logistic regression analysis was conducted. The discrete levels of each index were treated as distinct categories. Third, the model performance of the JCS score was assessed using the AUC for discrimination and a calibration plot and the HosmerLemeshow test for calibration. We performed a sensitivity analysis using the $\mathrm{M}$ component of the GCS score as a reference test instead of the total GCS score because some studies implied that the $\mathrm{M}$ component of GCS is a good predictor of outcomes. ${ }^{24}$

Continuous variables are expressed as median and interquartile range (IQR) values, while ordinal and categorical variables are expressed as frequencies and percentages. We used complete cases for all comparisons in the study analyses. All tests of significance were two-tailed, and a $\mathrm{p}$ value $<0.05$ was considered significant. Variables were analyzed using Stata V.15 (StataCorp, College Station, Texas, USA).

\section{RESULTS}

A total of 233104 patients were enrolled during the observation period; 224059 patients were excluded based on age or lack of information regarding the evaluation of consciousness and mortality (figure 1). Thus, a total of 9045 pediatric patients were finally included in the study. Most of the children were of school going age or older, and more than half were alert on arrival at the hospital. Approximately $70 \%$ of the patients were boys. Hypotension on arrival at the hospital was rare. The in-hospital mortality rate was $1.8 \%$ (table 1 ).

Figure 2 shows the AUCs of the GCS and JCS for model A (figure 2A) and model B (figure 2B). Figure 3 shows the results of the Hosmer-Lemeshow test. Because many children had a GCS score of 15 or a JCS 'alert' grade, their scores were combined and categorized into five groups for the Hosmer-Lemeshow test. Every point in each group lies on an approximately diagonal line.

The AUC of the GCS was 0.929 (95\% CI 0.904 to 0.954 ) in model A (figure 2A) and 0.975 (95\% CI 0.963 to 0.987 ) in model B (figure $2 \mathrm{~B}$ ). The HosmerLemeshow $\chi^{2}$ statistic was $0.00(p=1.00)$ in model A (figure 3A, online supplemental table 1 ) and was 4.14 $(\mathrm{p}=0.84)$ in model B (figure 3C, online supplemental table 2).

The AUC of the JCS was 0.930 (95\% CI 0.906 to 0.954 ) in model A (figure 2A) and was 0.974 (95\% CI 0.963 to 0.985 ) in model B (figure $2 \mathrm{~B}$ ). The HosmerLemeshow $\chi^{2}$ statistic was $0.00(\mathrm{p}=1.00)$ in model $\mathrm{A}$ (figure 3B, online supplemental table 1 ) and was 8.55 $(\mathrm{p}=0.38)$ in model B (figure 3D, online supplemental 
table 2). The ORs for each variable are shown in online supplemental table 3 .

The sensitivity analyses showed results similar to those of our main analysis (online supplemental figures 1 and 2). The AUC of the M component of GCS was 0.913 (95\% CI 0.886 to 0.940 ) in model A (online supplemental figure 1A) and was 0.973 (95\% CI 0.961 to 0.984 ) in model B (online supplemental figure 1B). The Hosmer-Lemeshow $\chi^{2}$ statistic was $0.00(p=1.00)$ in model A (online supplemental figure $2 \mathrm{~A}$ ) and was 6.21 $(\mathrm{p}=0.62)$ in model $\mathrm{B}$ (online supplemental figure $2 \mathrm{~B}$ ).

\section{DISCUSSION}

In this study, we used a Japanese national trauma database to evaluate the usefulness of the JCS for predicting mortality in children. We found no clinically meaningful differences between the JCS and GCS in terms of discrimination and calibration for in-hospital mortality in pediatric trauma patients.

Similar to previous studies conducted among adults, ${ }^{15-17}$ our study showed that the usefulness of the JCS in predicting mortality was similar to that of the GCS. Although a previous study included children as a part of the targeted population, no study has yet revealed the usefulness of the JCS in a subset of children. $^{25}$

In contrast to the GCS score, the JCS score can be obtained without calculations involving multiple items. Thus, paramedics, nurses and some physicians in Japan prefer to use the JCS. The results of this study demonstrate the usefulness of the JCS in children, improve our understanding of the scale and clarify its predictive value for mortality. This is beneficial to paramedics and healthcare providers who use the JCS frequently.

The GCS has been used as a covariate and incorporated as a scoring factor in various studies in the fields of emergency medicine and intensive care. ${ }^{326-29} \mathrm{In}$ addition to its usefulness in clinical practice, the JCS, like the GCS, could be used as a covariate in future studies on causal inference.

\section{Strength and limitations}

The strength of this study is that we evaluated the JCS and GCS simultaneously; therefore, we could compare their scores directly.

This study had some limitations. First, only patients registered in the database were included in the study. However, we believe that this study covers the most severe pediatric trauma cases in Japan because approximately three-quarters of the tertiary care hospitals in Japan are registered with the JTDB. ${ }^{14}$ Second, the granularity of patient details was limited because a database was used for this study. A large prospective, exhaustive study is needed to address these limitations. Third, our database did not include the functional outcomes, which represent the morbidity. Therefore, we could only compare the usefulness of the scores in terms of mortality. Lastly, the model for the mild disturbance of consciousness category was unstable given the small number of patients, which could have affected the results. However, since the receiver operating characteristic and calibration plots showed consistent results, we believe that this issue does not significantly impact the findings of the study.

In conclusion, using a Japanese nationwide trauma database, we demonstrated that the JCS is as valid as the GCS for the prediction of mortality. The results suggest that the JCS is a useful and relevant tool for pediatric trauma care and future research.

Acknowledgements We would like to thank Editage (www.editage.com) for English language editing.

Contributors YE is the guarantor. YE, YT and AT contributed to conceptualization, formal analysis and writing - original draft. TK, KI, MT, SY and YI contributed to supervision. All the authors approved the final manuscript.

Funding This research was funded through the support of JSPS KAKENHI Grant Number 20 K09261.

Competing interests None declared.

Patient consent for publication Not applicable.

Ethics approval The study was approved by the the Institutional Review Board of the National Hospital Organization Mito Medical Centre. The need for informed consent was waived due to the anonymity of the research data.

Provenance and peer review Not commissioned; externally peer reviewed.

Data availability statement Data may be obtained from a third party and are not publicly available. Data supporting the findings of this study are available from Japan Trauma Data Bank (JTDB), but there are restrictions on the use of these data. These data were used under license for this study and are therefore not available to the public. However, upon reasonable request, the data may be obtained with the permission of JTDB.

Open access This is an open access article distributed in accordance with the Creative Commons Attribution Non Commercial (CC BY-NC 4.0) license, which permits others to distribute, remix, adapt, build upon this work non-commercially, and license their derivative works on different terms, provided the original work is properly cited, appropriate credit is given, any changes made indicated, and the use is non-commercial. See: http://creativecommons.org/licenses/by-nc/4.0/.

ORCID iD

Yuki Enomoto http://orcid.org/0000-0002-3052-8602

\section{REFERENCES}

1 Teasdale G, Jennett B. Assessment of coma and impaired consciousness. A practical scale. Lancet 1974;2:81-4.

2 Teasdale G, Maas A, Lecky F, et al. The Glasgow coma scale at 40 years: standing the test of time. Lancet Neurol 2014;13:844-54.

3 Boyd CR, Tolson MA, Copes WS. Evaluating trauma care: the TRISS method. J Trauma Acute Care Surg 1987;27:370-8.

4 Thompson DO, Hurtado TR, Liao MM, et al. Validation of the simplified motor score in the out-of-hospital setting for the prediction of outcomes after traumatic brain injury. Ann Emerg Med 2011;58:417-25.

5 Brown JB, Forsythe RM, Stassen NA, et al. Evidence-based improvement of the National trauma triage protocol: the Glasgow coma scale versus Glasgow coma scale motor subscale. J Trauma Acute Care Surg 2014;77:95-102.

6 Eichelberger MR, Bowman LM, Sacco WJ, et al. Trauma score versus revised trauma score in TRISS to predict outcome in children with blunt trauma. Ann Emerg Med 1989;18:939-42.

7 Heather NL, Derraik JGB, Beca J, et al. Glasgow coma scale and outcomes after structural traumatic head injury in early childhood. PLoS One 2013;8:e82245.

8 Davis AL, Hochstadter E, Daya T, et al. The base deficit, international normalized ratio, and Glasgow coma scale (big) score, and functional outcome at hospital discharge in children with traumatic brain injury. Pediatr Crit Care Med 2019;20:970-9. 
9 Ohta T, Waga S, Handa W, et al. [New grading of level of disordered consiousness (author's transl)]. No Shinkei Geka 1974;2:623-7.

10 Enomoto Y, Tsuchiya A, Tsutsumi Y, et al. Association between physician-staffed helicopter versus ground emergency medica services and mortality for pediatric trauma patients: a retrospective nationwide cohort study. PLoS One 2020;15:e0237192.

11 Tada H, Takanashi J-ichi, Okuno H, et al. Predictive score for early diagnosis of acute encephalopathy with biphasic seizures and late reduced diffusion (AESD). J Neurol Sci 2015;358:62-5.

12 Tagami T, Matsui $\mathrm{H}$, Horiguchi $\mathrm{H}$, et al. Antithrombin and mortality in severe pneumonia patients with sepsis-associated disseminated intravascular coagulation: an observational nationwide study. $J$ Thromb Haemost 2014;12:1470-9.

13 Hatachi T, Michihata N, Inata Y, et al. Prognostic factors among children with acute encephalitis/encephalopathy associated with viral and other pathogens. Clin Infect Dis 2021;73:76-82.

14 Japan Trauma Care and Research. Japan trauma data bank report, 2018. Available: https://www.jtcr-jatec.org/traumabank/dataroom/ data/JTDB2018e.pdf [Accessed 5 Nov 2020].

15 Shigematsu K, Nakano $\mathrm{H}$, Watanabe $\mathrm{Y}$. The eye response test alone is sufficient to predict stroke outcome--reintroduction of Japan Coma Scale: a cohort study. BMJ Open 2013;3:e002736.

16 Yumoto T, Naito H, Yorifuji T, et al. Association of Japan coma scale score on hospital arrival with in-hospital mortality among trauma patients. BMC Emerg Med 2019;19:65.

17 Okada Y, Kiguchi T, liduka R, et al. Association between the Japan coma scale scores at the scene of injury and in-hospital outcomes in trauma patients: an analysis from the nationwide trauma database in Japan. BMJ Open 2019;9:e029706.

18 Ono K, Wada K, Takahara T, et al. Indications for computed tomography in patients with mild head injury. Neurol Med Chir 2007;47:291-8.

19 Gotoh O, Tamura A, Yasui N, et al. [Japan coma scale in the prediction of outcome after early surgery for aneurysmal subarachnoid hemorrhage]. No To Shinkei 1995;47:49-55.
20 Sakamoto Y. Syouni Shinkei Shindangaku (Japanese). Tokyo: Kinbara, 1978.

21 Borgialli DA, Mahajan P, Hoyle JD, et al. Performance of the pediatric Glasgow coma scale score in the evaluation of children with blunt head trauma. Acad Emerg Med 2016;23:878-84.

22 American Heart Association Inc. Pediatric advanced life support. 1st ed. Dallas, TX: American Heart Association Inc, 2017.

23 Fischer JE, Bachmann LM, Jaeschke R. A readers' guide to the interpretation of diagnostic test properties: clinical example of sepsis. Intensive Care Med 2003;29:1043-51.

24 Kupas DF, Melnychuk EM, Young AJ. Glasgow Coma Scale Motor Component ("Patient Does Not Follow Commands") Performs Similarly to Total Glasgow Coma Scale in Predicting Severe Injury in Trauma Patients. Ann Emerg Med 2016;68:744-50.

25 Yamagami K, Kurogi R, Kurogi A, et al. The influence of age on the outcomes of traumatic brain injury: findings from a Japanese nationwide survey (J-ASPECT Study-Traumatic brain injury). World Neurosurg 2019;130:e26-46.

26 Lecky F, Woodford M, Edwards A, et al. Trauma scoring systems and databases. Br J Anaesth 2014;113:286-94.

27 Haider AH, Hashmi ZG, Zafar SN, et al. Developing best practices to study trauma outcomes in large databases: an evidence-based approach to determine the best mortality risk adjustment model. $J$ Trauma Acute Care Surg 2014;76:1061-9.

28 Vincent JL, de Mendonça A, Cantraine F, et al. Use of the SOFA score to assess the incidence of organ dysfunction/failure in intensive care units: results of a multicenter, prospective study. Working group on "sepsis-related problems" of the European Society of Intensive Care Medicine. Crit Care Med 1998;26:1793-800.

29 Knaus WA, Draper EA, Wagner DP, et al. Apache II: a severity of disease classification system. Crit Care Med 1985;13:818-29. 